\title{
Observations of the intense and ultra-long burst GRB 041219a with the Germanium spectrometer on INTEGRAL ^
}

\author{
S. McBreen ${ }^{1,2}$, L. Hanlon ${ }^{3}$, S. McGlynn ${ }^{3}$, B. McBreen ${ }^{3}$, S. Foley ${ }^{3}$, R. Preece ${ }^{4}$, A. von Kienlin ${ }^{2}$, and O. R. Williams ${ }^{5}$ \\ ${ }^{1}$ Research and Scientific Support Department of ESA, ESTEC, Noordwijk, The Netherlands \\ e-mail: smcbreen@mpe.mpg.de \\ 2 Max-Planck-Institut für extraterrestrische Physik, 85748 Garching, Germany \\ 3 School of Physics, University College Dublin, Dublin 4, Ireland \\ ${ }^{4}$ Department of Physics, University of Alabama at Huntsville, USA \\ 5 ISOC, ESA/ESAC, Urb. Villafranca del Castillo, Madrid, Spain \\ Received 14 March 2006 / Accepted 18 April 2006
}

\section{ABSTRACT}

\begin{abstract}
Context. GRB 041219a is the brightest burst localised by INTEGRAL. The peak flux of $43 \mathrm{ph} \mathrm{cm}^{-2} \mathrm{~s}^{-1}\left(1.84 \times 10^{-5} \mathrm{erg} \mathrm{cm}^{-2} \mathrm{~s}^{-1}\right.$, $20 \mathrm{keV}-8 \mathrm{MeV}, 1 \mathrm{~s}$ integration) is greater than that for $\sim 98 \%$ of all bursts and the $T_{90}$ duration of $\sim 186 \mathrm{~s}(\sim 20 \mathrm{keV}-8 \mathrm{MeV})$ is longer than all but a small number of bursts. The intense burst occurred about $\sim 250 \mathrm{~s}$ after the precursor and the long delay enabled optical and near infrared telescopes to observe the prompt emission.

Aims. We present comprehensive results of the temporal and spectral analyses, including line and afterglow searches using the spectrometer, SPI, aboard INTEGRAL, BAT on Swift and ASM on Rossi X-ray Timing Explorer. We avail of multi-wavelength data to generate broadband spectra of GRB 041219a and afterglow.

Methods. Spectra for the burst and sub-intervals were fit by the Band model and also by the quasithermal model. The high resolution Germanium spectrometer data were searched for emission and absorption features and for $\gamma$-ray afterglow.

Results. The overall burst and sub-intervals are well fit by the Band model. The photon index below the break energy shows a marked change after the quiescent time interval. In addition the spectra are well described by a black body component with a power law. The burst was detected by BAT and ASM during the long quiescent interval in SPI indicating the central engine might not be dormant but that the emission occurs in different bands. No significant emission or absorption features were found and limits of $900 \mathrm{eV}$ and $120 \mathrm{eV}$ are set on the most significant features. No $\gamma$-ray afterglow was detected from the end of the prompt phase to $\sim 12 \mathrm{~h}$ post-burst. Broadband spectra of the prompt emission were generated in 7 time intervals using $\gamma$-ray, X-ray, optical and near-infrared data and these were compared to the high-redshift burst GRB 050904. The optical and $\gamma$-ray emission are correlated in GRB 041219a. We estimate isotropic radiated energy $\left(E_{\text {iso }}\right)$ to be $\sim 5 \times 10^{52} \mathrm{erg}$. The spectral lag was determined using data from the BAT and it changes throughout the burst. A number of pseudo-redshifts were evaluated and large dispersion in values was found.
\end{abstract}

Key words. gamma-rays: bursts - gamma-rays: observations

\section{Introduction}

The afterglow era of gamma-ray bursts (GRBs) has yielded many discoveries, in particular, conclusive proof of the cosmological origin and association with supernovae (e.g. Costa et al. 1997; Hjorth et al. 2003). INTEGRAL has detected 37 GRBs to date most of which were quite weak including a member of the low-luminosity class of bursts GRB 031203 (Sazonov et al. 2004; Watson et al. 2004) and an X-ray rich burst GRB 040223 (McGlynn et al. 2005). For recent reviews of $\gamma$-ray bursts see Zhang \& Mészáros (2004) and Piran (2004). In this paper we present the results of the $\gamma$-ray spectral and temporal characteristics of the burst GRB 041219a obtained with the high resolution spectrometer, SPI, aboard INTEGRAL (Winkler et al. 2003). SPI is a coded-mask telescope with a fully coded field of view of $16^{\circ}$ that uses a 19 pixel high spectral resolution $\mathrm{Ge}$ detector. The detectors cover the energy range $20 \mathrm{keV}-8 \mathrm{MeV}$ with an energy resolution of $2-8 \mathrm{keV}$ FWHM.

^ Based on observations with INTEGRAL, an ESA project with instruments and science data centre funded by ESA member states (especially the PI countries: Denmark, France, Germany, Italy, Switzerland, Spain), Czech Republic and Poland, and with the participation of Russia and the USA.
A detailed description of SPI can be found in Vedrenne et al. (2003). Data from the imager IBIS is not included here because proprietary rights were awarded to other groups. Analysis of the light curves observed by Burst Alert Telescope (BAT) on Swift in the range 15-350 keV (Gehrels et al. 2004) and the Rossi X-ray Timing Explorer All Sky Monitor (ASM) in the energy range 1.5-12 keV (Levine et al. 1996) are also presented. GRB 041219a is the brightest burst detected by the INTEGRAL burst alert system (IBAS) (Mereghetti et al. 2003) and prompt emission was detected in the optical (Vestrand et al. 2005) and near infrared (Blake et al. 2005).

\section{Observations}

GRB 041219a was detected by IBAS at 01:42:18 UTC on December 19th 2004 (Götz et al. 2004) at a location of right ascension $00^{\mathrm{h}} 24^{\mathrm{m}} 25.8^{\mathrm{s}}$, declination $+62^{\circ} 50^{\prime} 05.6^{\prime \prime}$ (Galactic latitude and longitude of $0.12^{\circ}, 119.85^{\circ}$ ) at a detector off-axis angle of $3.2^{\circ}$. GRB 041219a was also detected by BAT (Barthelmy et al. 2004) at a location consistent with the IBAS position. Levine \& Remillard (2004) reported a serendipitous observation of GRB 041219a during two $90 \mathrm{~s}$ dwell times with the Rossi $X$-ray Timing Explorer All Sky Monitor (ASM) in the energy 

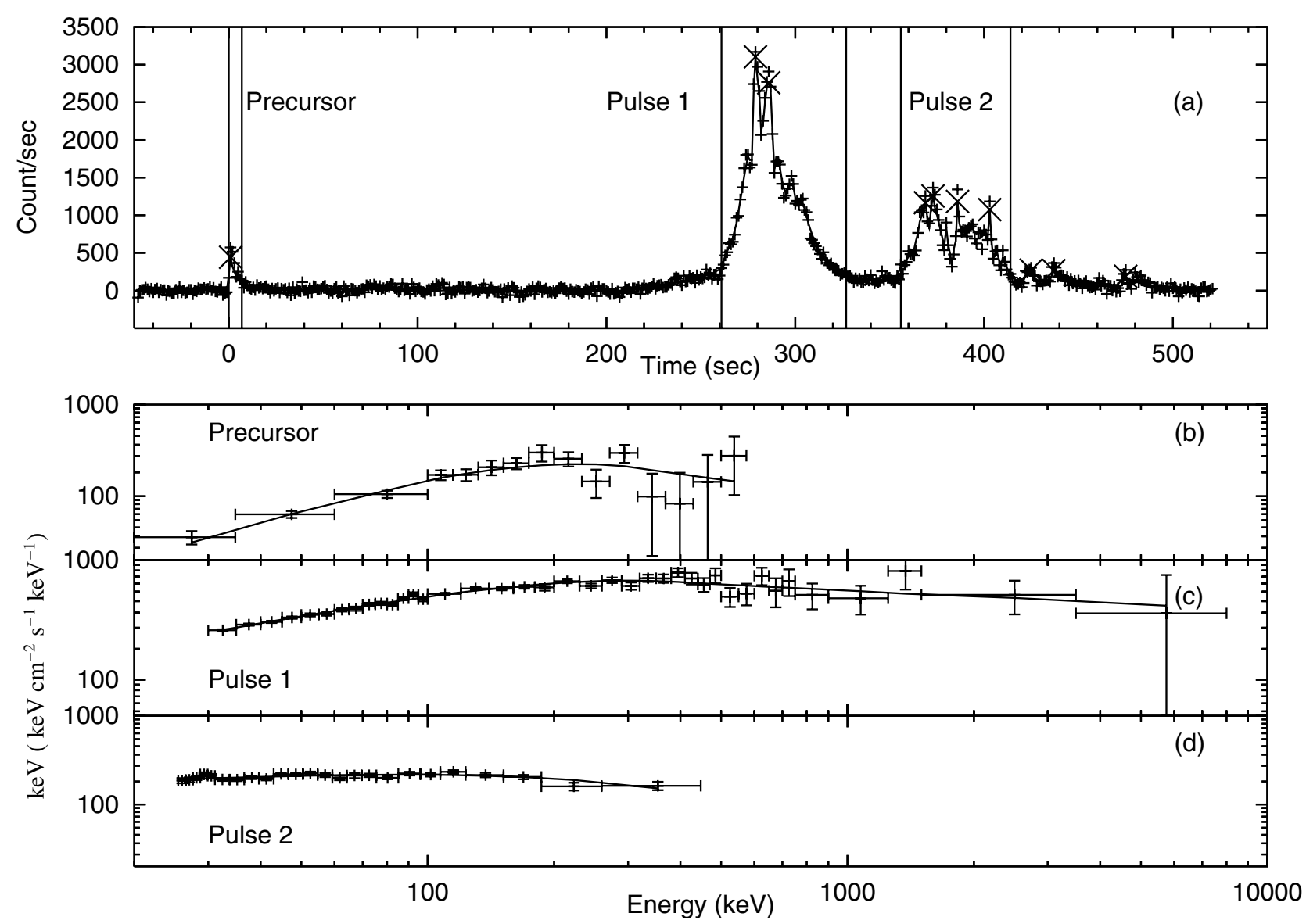

Fig. 1. SPI light curves and spectra of GRB 041219a. a) denoised and background subtracted light curve where significant pulses selected by the algorithm are marked with an $x$. The three marked sections denote the time intervals over which the spectra were analysed. The start and end times of the intervals and the parameters of best fit Band spectra are presented in Table 1. Spectrum and Band model fits for b) the precursor pulse $(0-7 \mathrm{~s}) ; \mathbf{c})$ pulse $1(261-327 \mathrm{~s})$; d) pulse $2(356-414 \mathrm{~s})$.

range 1.5-12 keV (Levine et al. 1996). The burst was in the field of view from $6 \mathrm{~s}$ after the trigger for 3 min apart from a $6 \mathrm{~s}$ gap. Prompt optical emission correlated with the $\gamma$-ray emission was reported by Vestrand et al. (2005). Infrared emission was detected $7.2 \mathrm{~min}$ after the burst trigger at the tail end of the prompt $\gamma$-ray emission (Blake et al. 2005). Radio observations detected a source at $8.5 \mathrm{GHz}$ at $T_{0}+1.1$ days (Soderberg \& Frail 2004) and two observations at $4.9 \mathrm{GHz}$ beginning at $T_{0}+1.59$ days (van der Horst et al. 2004a) and $T_{0}+2.54$ by (van der Horst et al. 2004b) reported an increased flux density in the second epoch. There is no measured redshift available for this burst.

\section{Analysis and results}

\subsection{Light curves}

The SPI light curve in the broad energy band from $20 \mathrm{keV}-8 \mathrm{MeV}$ at $1 \mathrm{~s}$ resolution is given in Fig. 1a. The light curves are generated from housekeeping data (Moran et al. 2005). A section of the light curve detected by the BAT is shown in Fig. 2 using the data from Fenimore et al. (2004) and no spectral information is available. The $T_{90}$ value of $186 \mathrm{~s}$ ( $\sim 20 \mathrm{keV}-8 \mathrm{MeV}$ ) was determined from the SPI light curve. $T_{90}$ is the time for 5 to $95 \%$ of the flux to be accumulated and the values are shorter than the burst duration in this case because of the quiescent interval and the precursor has only $\sim 2 \%$ of the counts.

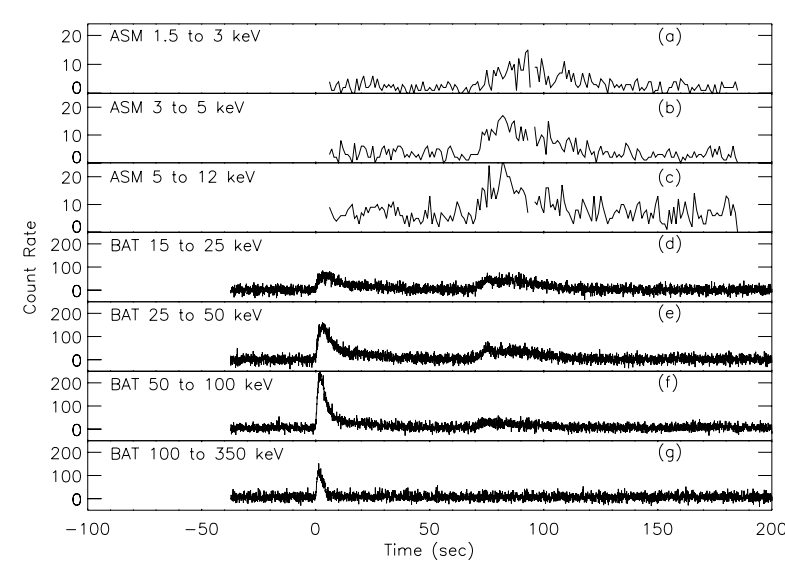

Fig. 2. Rossi ASM a)-c) and Swift BAT d)-g) light curves of GRB 041219a up to $T_{0}+200 \mathrm{~s}$. Rossi ASM data are available only for two $90 \mathrm{~s}$ dwell times starting at $T_{0}+6 \mathrm{~s}$ and $T_{0}+96 \mathrm{~s}$. The ASM and BAT light curves have emission in the interval at about $80 \mathrm{~s}$ that is quiescent in the SPI light curve (Fig. 1). The Rossi-ASM data were obtained courtesy of Alan Levine.

The temporal structure of the burst is unusual, with an initial weak precursor pulse followed by a long quiescent time interval and the main emission beginning at $\sim 250 \mathrm{~s}$ post-trigger (Fig. 1a). The SPI light curve was denoised using a wavelet 
Table 1. Spectral properties of GRB 041219a. The columns refer to the emission region, time interval of spectral fits, low-energy power-law index

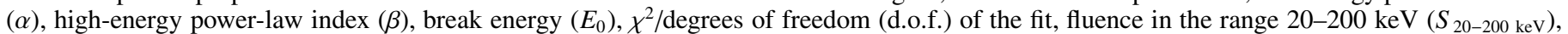
fluence in the range $20 \mathrm{keV}-8 \mathrm{MeV}\left(S_{20} \mathrm{keV}-8 \mathrm{MeV}\right)$, and value of the spectral lag between BAT channels 1 and 3 over the specified interval. Errors on spectral parameters quoted for a $90 \%$ confidence level and the fit parameters are for the spectra shown in Fig. 1.

\begin{tabular}{|c|c|c|c|c|c|c|c|c|}
\hline $\begin{array}{l}\text { Emission } \\
\text { region }\end{array}$ & Time (s) & $\alpha$ & $\beta$ & $\begin{array}{c}E_{0} \\
(\mathrm{keV})\end{array}$ & $\chi^{2} /$ d.o.f. & $\begin{array}{l}S_{20-200 \mathrm{keV}} \\
\left(\mathrm{erg} \mathrm{cm}^{-2}\right)\end{array}$ & $\begin{array}{c}S_{20 \mathrm{keV}-8 \mathrm{MeV}} \\
\left(\mathrm{erg} \mathrm{cm}^{-2}\right)\end{array}$ & Lag (s) \\
\hline Precursor & $0-7$ & $-0.45_{-0.30}^{+0.37}$ & $-2.62_{-74}^{+0.73}$ & $145.4_{-48.7}^{+79.7}$ & $12.1 / 11$ & $2.8 \times 10^{-6}$ & $7.1 \times 10^{-6}$ & $1.2 \pm 0.08$ \\
\hline 1st Pulse & $261-327$ & $-1.5_{-0.06}^{+0.08}$ & $-1.95_{-0.21}^{+0.08}$ & $568.3_{-205.2}^{-48.1}$ & $57.5 / 50$ & $7.0 \times 10^{-5}$ & $2.6 \times 10^{-4}$ & $0.11 \mathrm{~s} \pm 0.01$ \\
\hline 2nd Pulse & $356-414$ & $-1.76_{-0.08}^{+0.06}$ & $-3^{-0.21}$ & $363.6_{-995}^{-195.1}$ & $30.9 / 21$ & $4.0 \times 10^{-5}$ & $1.0 \times 10^{-4}$ & $0.15 \pm 0.01$ \\
\hline 1st \& 2nd Pulse & $261-414$ & $-1.43_{-0.06}^{+0.08}$ & $-2.06_{-0.12}^{+0.09}$ & $299.2_{-73.9}^{-995.2}$ & $16.5 / 25$ & $1.3 \times 10^{-4}$ & $3.7 \times 10^{-4}$ & $0.17 \pm 0.02$ \\
\hline Burst & $0-580$ & $-1.48_{-0.07}^{+0.06}$ & $-1.92_{-0.13}^{+0.07}$ & $365.9_{-108.2}^{+191.9}$ & $51.9 / 30$ & $1.6 \times 10^{-4}$ & $5.7 \times 10^{-4}$ & \\
\hline
\end{tabular}

${ }^{\dagger} \beta$ value frozen at -3 .

Table 2. Spectral fits to GRB 041219a using the quasithermal model. The columns refer to the emission region, time interval of spectral fits, $k T$, power-law index $(\Gamma), \chi^{2} /$ degrees of freedom (d.o.f.) of the fit. Errors on spectral parameters quoted for a $90 \%$ confidence level.

\begin{tabular}{ccccccc}
\hline \hline $\begin{array}{c}\text { Emission } \\
\text { region }\end{array}$ & Time (s) & $k T$ & $\Gamma$ & $\chi^{2} /$ d.o.f. & $\begin{array}{c}S_{20-200 \mathrm{keV} \text { in the blackbody }} \\
\text { component }\left(\mathrm{erg} \mathrm{cm}^{-2} \mathrm{~s}^{-1}\right)\end{array}$ & $\begin{array}{c}\text { \% Flux in the blackbody } \\
\text { component }(20-200 \mathrm{keV})\end{array}$ \\
\hline Precursor & $0-7$ & $45.7_{-8.7}^{+9.1}$ & $1.58_{-0.28}^{+0.53}$ & $9.25 / 11$ & $1.4 \times 10^{-6}$ & $49 \%$ \\
1st Pulse & $261-327$ & $35.1_{-4.7}^{+5.5}$ & $1.74_{-0.02}^{+0.02}$ & $68 / 50$ & $8.7 \times 10^{-6}$ & $11.6 \%$ \\
2nd Pulse & $356-414$ & $27.3_{-4.8}^{+4.9}$ & $2.1_{-0.08}^{+0.70}$ & $28.9 / 21$ & $5.8 \times 10^{-6}$ & $12.7 \%$ \\
\hline
\end{tabular}

analysis (Quilligan et al. 2002) and the pulses selected by a pulse decomposition algorithm are shown in Fig. 1a.

Although the SPI light curve is quiescent from $\sim 7-200 \mathrm{~s}$, emission is detected in the BAT light curve particularly in the lower energy channels 1 and 2 (Fig. 2) (15-25 keV and $25-50 \mathrm{keV}$ ) (Fenimore et al. 2004). In addition a spectrally soft pulse was detected in the ASM (Levine \& Remillard 2004) beginning at $80 \mathrm{~s}$ in the quiescent period of the SPI observation (Fig. 2). The value of $T_{90}$ depends very much on the bandwidth over which it is measured.

The spectral lags and associated errors were measured between BAT channels 1 and 3 using the cross-correlation technique described in Norris et al. (2000). The code was validated by comparing results for a test case GRB obtained by Norris et al. and consistency within the errors was obtained, over a range of parameters. The values for the lag in Table 1 (Col. 9) were measured for regions where the intensity was greater than $0.1 \times$ peak intensity of all channels combined. The timing data were oversampled by a factor of 10 for the main emission and a factor of 4 for the precursor in order to allow bootstrap errors to be calculated. The maximum of the cubic fit to the crosscorrelation function was used as the measure of the spectral lag. The lag in the precursor is much longer than that in the remainder of the burst (Table 1).

\subsection{Spectral analysis}

The spectrum of GRB 041219a was extracted using Online Software Analysis version 5.0 available from the INTEGRAL Data Science Centre ${ }^{1}$ (see also Diehl et al 2003).GRB 041219a is the brightest burst localised by INTEGRAL to date. Its peak flux of $43 \mathrm{ph} \mathrm{cm}^{-2} \mathrm{~s}^{-1}\left(1.84 \times 10^{-5} \mathrm{erg} \mathrm{cm}^{-2} \mathrm{~s}^{-1}\right)(20 \mathrm{keV}-8 \mathrm{MeV}$, $1 \mathrm{~s}$ integration) is greater than $\sim 98 \%$ of bursts detected by BATSE while its $T_{90}$ duration of $186 \mathrm{~s}(\sim 20 \mathrm{keV}-8 \mathrm{MeV})$ is longer than all but a handful ( 4\%) of BATSE GRBs (Paciesas et al. 1999). The spectrum of the burst and sub-intervals are well

\footnotetext{
${ }^{1}$ http://isdc.unige.ch/
}

fit by the Band model (Band et al. 1993) and the parameters are presented in Fig. 1 and Table 1 (Cols. 1-8). The parameters of the spectrum evolve during the burst. For instance $\alpha$ is remarkably higher in the initial pulse in comparison to the main emission phase. The value of $\alpha$ for the main emission phase is $-1.43_{-0.06}^{+0.08}$ and is used to derive the redshift in Sect. 4.5. The peak energy, $E_{\text {peak }}$, is given by $(2+\alpha) \times E_{0}$ and the evolution of $E_{\text {peak }}$ shows softening in the main emission phase. The value of $E_{\text {peak }}$ in the interval of the main emission phase is $170_{-42.6}^{+44.5} \mathrm{keV}$ and this value is used to derive the radiated and isotropic energy in Sect. 4.5.

Ryde (2005b) suggested that thermal emission may be ubiquitous in GRBs and fit a black body + power law model to a number of bright BATSE bursts. Ryde (2005b) reported that the temperature was intially constant or weakly decreasing with a shallow power law and that the power law index steepened throughout the burst. We fit the quasithermal model to GRB 041219a and the results are shown in Table 2 and Fig. 3. In this interpretation the black body contributes more strongly in the precursor than in pulses 1 and 2 .

\subsection{Spectral lines}

A search for line emission in the SPI data was carried out on the brightest pulse in the burst. The search involved finding the best continuum model for the data and then adding a Gaussian emission line of varying width ( $1 \mathrm{keV}$ to $20 \mathrm{keV})$ to the model at energies from $30 \mathrm{keV}$ to $1 \mathrm{MeV}$. The F-test was used to evaluate the resulting improvement in the fit for each line width and each energy. However, it is well known that the F-test alone cannot be used to check for the presence of a line (Protassov et al. 2002) and must be calibrated for false positives. We adopt a similar approach to that suggested by Protassov et al. (2002). The spectrum for which the largest F-test value was obtained was chosen and Monte Carlo simulations were carried out to test the number of times a more significant F-test value would be detected in simulated data of the continuum model. A large number of spectra 


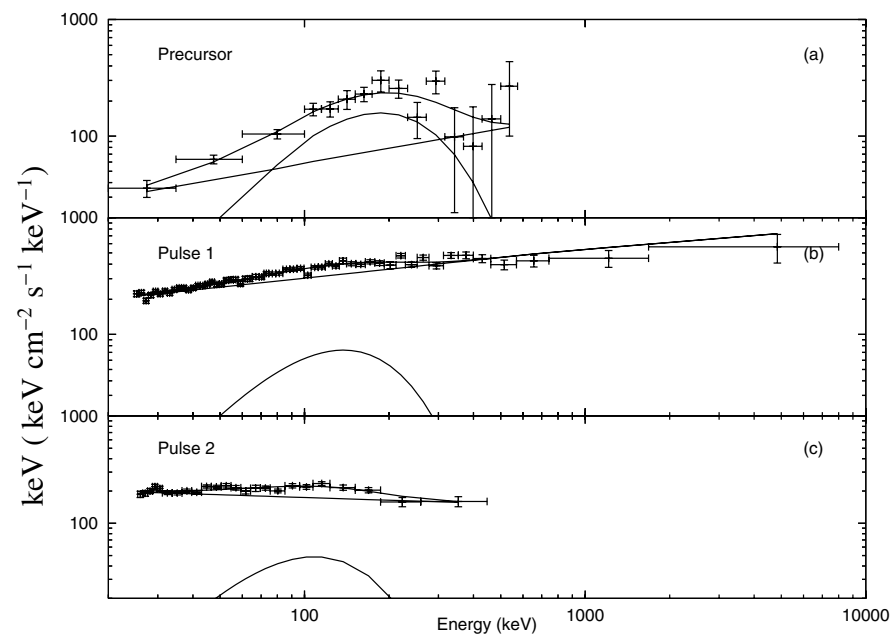

Fig. 3. Spectral fits to a) the precursor, b) pulse 1 and c) pulse 2 of GRB 041219a using combined black body and power law fits. The data are shown along with the combined fit and the black body and power law components.

(10000) of the continuum were generated. The Band model and Band model+ emission line were fit to each generated spectrum and the F-test for the improvement in the fit was recorded for each. The same approach was adopted to search for absorption features. The false positive rate was evaluated.

The most significant emission line feature was $4 \mathrm{keV}$ wide at $89 \mathrm{keV}$ with an F-test value of 4.6. Of the 10000 simulated spectra, 5850 resulted in an improvement in $\chi^{2}$ and out of this fraction $5.2 \%$ of trials resulted in a higher value of F-test than 4.6. The equivalent width of this emission line is $900 \mathrm{eV}$ $\left(1.4 \times 10^{-9} \mathrm{erg}\right)$.

The most significant absorption feature had a width of $3 \mathrm{keV}$ at $103 \mathrm{keV}$ with an F-test value of 7.8. Of the 10000 simulations that were run, 5876 resulted in an improvement in $\chi^{2}$ and $2.5 \%$ of these produced an F-test value higher than 7.8. The equivalent width of this absorption feature is $120 \mathrm{eV}\left(1.9 \times 10^{-10} \mathrm{erg}\right)$.

No significant emission or absorption features were found.

\subsection{Afterglow search with SPI}

A search for a $\gamma$-ray afterglow was performed on the available data for up to $12 \mathrm{~h}$ after the burst trigger. The data was split into three separate time intervals and a spectrum extracted. Significant emission from the burst position was not detected even when the data were summed over the entire $12 \mathrm{~h}$. The $3 \sigma$ upper limits for the subsequent time intervals are given in Fig. 4.

\subsection{Broadband spectra}

The broadband spectra of the prompt emission for the GRB 041219a are plotted in 7 time intervals using a combination of $\gamma$-ray data from SPI and BAT, X-ray data from ASM, optical data from Vestrand et al. (2005) and infrared data from Blake et al. (2005) corrected for extinction. Figure 5 shows three spectra during the initial $\sim 120 \mathrm{~s}$ generated from Rossi-ASM, SwiftBAT and INTEGRAL-SPI data in three different time intervals.

The broadband spectra of the prompt emission for GRB 041219a are given in Fig. 6 for the four time intervals of simultaneous optical and gamma observations. In the case of the interval (D) there are also simultaneous near infrared results from PARITEL (Blake et al. 2005). The broadband spectra

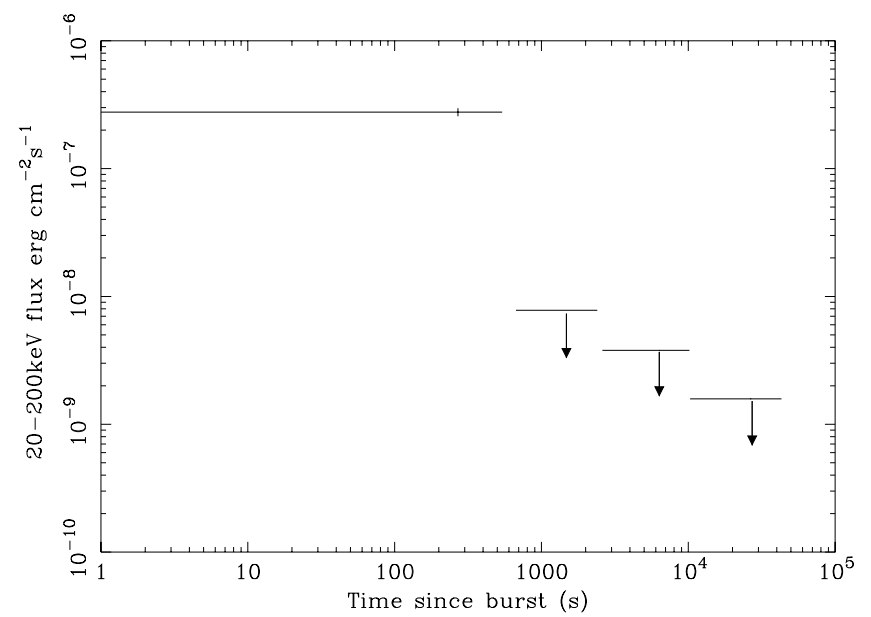

Fig. 4. The flux averaged over the burst duration of GRB 041219a and $3 \sigma$ upper limits in the energy range $20-200 \mathrm{keV}$. The arrows give the $3 \sigma$ limits over the time $670 \mathrm{~s}$ to $43000 \mathrm{~s}$ after the burst.

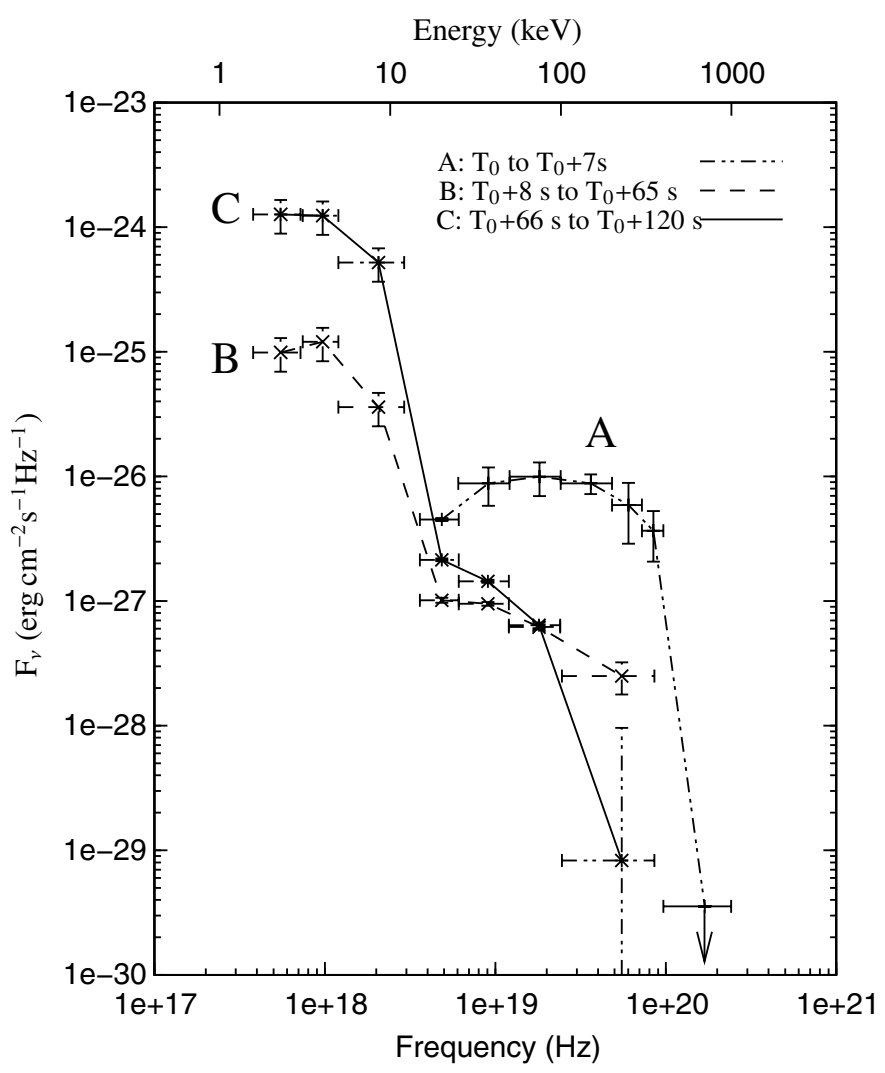

Fig. 5. Broadband spectra of the initial 120 s of GRB 041219a in the $\mathrm{X}$-ray and gamma ray region. The data are plotted in flux density (erg cm ${ }^{-1} \mathrm{~s}^{-1} \mathrm{~Hz}^{-1}$ ) versus observed frequency (lower axis) and $\mathrm{keV}$ (upper axis). The first interval $\left(T_{0}\right.$ to $\left.T_{0}+7 \mathrm{~s}\right)$ includes the precursor pulse and the third interval $T_{0}+8 \mathrm{~s}$ to $T_{0}+66 \mathrm{~s}$ includes the soft pulse in the long quiescent interval. The broad spectra were generated using ASM X-ray data and $\gamma$-ray data from BAT and SPI.

of GRB 041219a were originally presented by Vestrand et al. (2005). The energy range has been expanded here by including the SPI results for intervals (A) to (D) and near infrared results for interval (D). The broadband spectrum of the afterglow from GRB 041219a is shown $12 \mathrm{~h}$ after the burst (E) using infrared and optical data from Blake et al. (2005) and Sonoda et al. (2004) respectively and an upper limit from SPI. The simultaneous 
optical, X-ray (Boër et al. 2006) and gamma ray results for the high redshift GRB 050904 are shown in the interval 151 to $254 \mathrm{~s}$ for comparison.

\section{Discussion}

\subsection{Spectral behaviour of GRB $041219 a$}

In most GRB models, the main event is preceded by less intense emission, characterised by a thermal spectrum, called a precursor. Precursors are associated with the transition of the fireball from an optically thick to an optically thin environment (e.g. Piran 2004; Ramirez-Ruiz et al. 2002). A rigorous definition of precursors is problematic because of the complexity of GRB temporal structures. Koshut et al. (1995) defined precursors that had a peak intensity lower than that of the whole burst and were followed by a period of quiescence longer than the remaining duration of the burst. Soft precursors, occurring before the trigger, have been detected by many instruments e.g. Ginga (Murakami et al. 1991), HETE (Vanderspek et al. 2004), and BATSE (e.g. Lazzati 2005). The Ginga precursor has a thermal spectrum and has been compared with theoretical expectations (Ramirez-Ruiz et al. 2002). The precursor pulse was fit by a Band model and a black blody+power law model and is quite different from the main emission. The properties of the precursor spectrum are shown in Tables 1 and 2 respectively.

The $\beta$ values for a simple cooling spectrum and an instantaneous spectrum, in which cooling effects are negligible, are $-\frac{(p+2)}{2}$ and $-\frac{(p+1)}{2}$ respectively, where $p$ is the electron index. From Table 1, using the well constrained $\beta$ values for the main emission phase, the derived $p$ values are $2.12_{-0.18}^{+0.24}$ and $3.12_{-0.24}^{+0.18}$.

The value of $\alpha$ changes significantly between the precursor and main emission phase. $\alpha$ is predicted by the synchrotron shock model to be between $-3 / 2$ and $-1 / 2$, although taking into account non-isotropic electron pitch angles and/or selfabsorption can produce $\alpha$ values up to and above 0 (Lloyd \& Petrosian 2000). The evolution of $\alpha$ may be evidence of changes in opacity or electron pitch angle from pulse to pulse. There is a tendency for low break energies to give harder $\alpha$ values, since the spectrum does not reach the asymptotic value in the limited spectral fitting window.

The majority of GRBs have low energy power law spectral indices in the range $-3 / 2<\alpha<-2 / 3$ (e.g. Crider et al. 1997; Preece et al. 2000; Ghirlanda et al. 2002). However a substantial fraction of GRBs have $\alpha>-2 / 3$ and lie outside the optically thin synchrotron model (Preece et al. 2002). A number of explanations have been offered such as jitter radiation (Medvedev 2000), synchrotron emission from particles with an anisotropic pitch angle (Lloyd \& Petrosian 2000; Lloyd-Ronning \& Ramirez-Ruiz 2002), synchrotron self-Compton or inverse Compton off photospheric photons (Mészáros \& Rees 2000), Compton drag (e.g. Lazzati et al. 2000), Comptonisation of low energy photons (e.g. Liang 1997).

A number of authors have discussed the possibility of fitting thermal components to time resolved or average GRB spectra (e.g. Ghirlanda et al. 2003; Kaneko et al. 2003; Ryde 2004, 2005b; Bosnjak et al. 2006). Ghirlanda et al. (2003) studied time resolved spectra of a number of BATSE bursts with very hard spectra and found that the spectra were not adequately explained by non-thermal emission models. In fact, they found that spectra early in the burst were well fit by a black body spectral model. We find an improvement in $\chi^{2}$ for the blackbody+power law model over the Band model for the precursor phase of GRB 041219a where $49 \%$ of the flux is contained in the blackbody component (Table 2). The Band model provides a better description of the continuum spectrum in pulse 1 and similar goodness of fit values are achieved for pulse 2 . The fits of the quasithermal model show that the value of $k T$ is highest for the precursor pulse and declines during the burst. Kaneko et al. (2003) reported BATSE-BeppoSAX joint analysis of GRB 970111 suggesting existence of a blackbody component with temperature $\sim 40-70 \mathrm{keV}$ in the first $5 \mathrm{~s}$ of this burst in agreement with GRB 041219a. Recently, Ryde (2005b) studied the prompt emission from 25 of the brightest pulses in the catalogue of Kocevski et al. (2003) and found that time resolved spectra were equally well fitted by the black body+power law model and with the Band model. In addition, Ryde (2005b) found that the thermal and power law components evolve together with the black body temperature decreasing with time. The spectrum of GRB 041219a can also be described by the quasithermal model (Table 2).

Recently, Rees \& Mészáros (2005) suggested that the $E_{\text {peak }}$ in the $\gamma$-ray spectrum is due to the Comptonized thermal component from the photosphere, where the comoving optical depth falls to unity. The thermal emission from a laminar and steady jet when viewed head-on, would give rise to a thermal spectrum peaking in the X-ray or $\gamma$-ray band. The resulting spectrum would be the superposition of the Comptonized thermal component and the power law from the synchrotron emission.

\subsection{Spectral lines}

The issue of narrow spectral lines in the prompt emission from GRBs has been controversial. The line features originally identified in spectra at $\sim 20-60 \mathrm{keV}$ (Mazets et al. 1980) were interpreted as cyclotron line emission from electrons in neutron star magnetic fields and were used to support the neutron star origin hypothesis for GRBs. The BATSE Spectroscopy Detectors were determined to be quite capable of detecting features (Band et al. 1995), such as had been reported by Ginga (Murakami et al. 1988), if they were a common occurance. The results from an automated line search of BATSE data, however, were inconclusive (Briggs et al. 1998). Our analysis revealed no significant emission or absorption features in GRB 041219a.

In the context of what is now understood about the progenitors and distances of GRBs, the explanation for narrow $\gamma$-ray spectral features in the prompt emission is necessarily different to the original hypothesis and may be much more complex. Nevertheless, spectral line features are an extremely powerful probe of the burst environment. This has been amply illustrated by the analyses and interpretations of transient X-ray Fe features and absorption edges in both the prompt emission and afterglows of some GRBs (see e.g. Böttcher 2004, for a review) which have been used to impose general physics constraints on the homogeneity, isotropy and location of the reprocessing material with respect to the burst source. No strong evidence for $\gamma$-ray emission or absorption features has been found in the brightest pulse of GRB 041219a.

\subsection{Temporal behaviour GRB $041219 a$}

GRB 041219a has an unusual time history because the initial pulse is followed by a long period of quiescence. However it should be noted that although the time interval is quiescent in the SPI light curve, emission is detected in the three Rossi-ASMand Swift-BAT light curves (Fig. 2) at $\sim T_{0}+80 \mathrm{~s}$. Similar light curves with a weak triggering pulse followed by 
a long quiescent time interval have been observed by BATSE (Fishman et al. 1994). The sample of Quilligan et al. (2002) contained 319 bright BATSE GRBs and two of the bursts show similar temporal evolution to GRB 041219a (triggers 6451 and 7575). In addition the $\gamma$-ray light curves of GRB 050820a (Page et al. 2005) and GRB 060124 (Holland et al. 2006a; Romano et al. 2006) show a similar weak precursor pulse and a long quiescent interval before the main pulse as noted by Pal'Shin \& Frederiks (2005) and Golenetskii et al. (2006).

Lazzati (2005) searched for "non-triggering" precursors in a sample of bright, long BATSE burst light curves and found that in $20 \%$ of cases there is evidence of emission above the background coming from the same direction as the GRB. This emission is characterized by a softer spectrum with respect to the burst spectrum and contains up to $1 \%$ of the counts and typically has a non-thermal power-law spectrum. The precursor type pulse in GRB 041219 a contains $2 \%$ of the total fluence $(50-300 \mathrm{keV})$ and is similar to precursors in the BATSE sample, except that it was a triggering event.

A number of studies have been made of periods of quiescence in GRBs. A trend was found between the duration of the quiescent time and duration of the following emission period (Ramirez-Ruiz \& Merloni 2001) that is consistent with GRB 041219a. Quilligan et al. (2002) showed that the measured distribution of time intervals between pulses in BATSE bursts is best fit by lognormal with allowance made for the excess in time intervals $>15$ s. The Pareto Lévy tail (Montroll \& Shlesinger 1982) of the time intervals is well fit by a power-law of slope -1 (Quilligan et al. 2002).

The model of a GRB as a relaxation system (McBreen et al. 2002a), which continuously accumulates energy and discontinuously releases it accounts for the correlated pulses properties and time intervals between pulses (McBreen et al. 2002b; Nakar $\&$ Piran 2002). This model can be extended to include periods of quiescence if the system returns to a more stable configuration that might be caused by a total release of accumulated energy. These general considerations do not identify the emission mechanism. Periods of quiescence can in principle, be caused by a modulated relativistic wind or a switching off of the central engine (Ramirez-Ruiz et al. 2001).

Drago \& Pagliara (2005) found that similarities between the pre- and post-emission periods suggest that both emission periods are produced by the same mechanism and that long quiescent intervals are generated by a switching off of the engine rather than a modulation of a continuous wind.

However, it should be noted that GRB 041219a was detected at lower energies by BAT and ASM during the long quiescent interval in SPI indicating the central engine might not be dormant but that the emission occurs in different bands.

\subsection{Spectral lags}

The results on the spectral lags in GRB 041219a are presented in Table 1. The spectral lags of GRBs and their evolution are vital tools for probing the emission mechanism, however their interpretation is not straightforward.

The empirical connection between the rate of spectral evolution and spectral lag should ultimately reveal the underlying physical mechanism for the lag-luminosity relation (Kocevski \& Liang 2003). The lag correlates linearly with the decay timescale of single pulse bursts and hence with the peak-energy decay (Ryde 2005a). This implies that the lag-luminosity relation should translate into one involving the pulse timescale and hence to the processes forming the pulse in the outflowing plasma. The distillation of the spectral lag into a combination of spectral and temporal pulse properties leads naturally to the conclusion that lag should evolve during the burst. Hakkila \& Giblin (2004) reported BATSE bursts with greater spectral lags after a long quiescent interval. Ryde et al. (2005) also reported lags differing considerably within bursts. These changing lags have been observed in a sample of BATSE bursts (Chen et al. 2005) but with no obvious variation in lag as a function of individual pulse luminosity. In the case of GRB 041219a, there is a relatively long lag in the precursor type pulse (Table 1), with much shorter lags determined in the main emission phase. The pulses are slower in the first half of the burst, split by the tallest pulse in the burst, using the BAT four channel data. The fact the lag is not constant throughout the burst has obvious consequences for its use a luminosity indictor.

\subsection{Constraints on redshift and energy}

Amati et al. (2002) derived a relationship between $\alpha$ and redshift from BeppoSAX $\gamma$-ray bursts with known redshifts which reflects a dependence of $\alpha$ on the $E_{\text {peak }}$. Using the value of $\alpha=-1.43_{-0.06}^{+0.08}$ from all sub-intervals in the main emission phase yields $z=1.43_{-0.38}^{+0.55}$. The relationship between $E_{\text {peak }}$ and $E_{\text {iso }}$ (Amati et al. 2002; Ghirlanda et al. 2004) can then be used to estimate the isotropic radiated energy of $E_{\text {iso }}$ of $\sim 5 \times 10^{52} \mathrm{erg}$.

Recently Pelangeon et al. (2005) reported a new pseudoredshift indicator, improving the original methods of Atteia (2003). We calculated the new pseudo-redshift using the ratio between the bolometric luminosity over the brightest $15 \mathrm{~s}$ and the observed $E_{\text {peak }}$. A value of $X=1.3$ was determined, which corresponds to a value of $z \sim 0.3$. We regard this value as a lower limit because $\alpha=-1.0$ was used for calibration and is very different from the value of $\alpha$ for the main emission peak of -1.5 . The method Yonetoku et al. (2004) yields a value of $z=0.7 \pm 0.3$ which is lower than than the Amati value but still consistent within the combined uncertainties. However there is clearly a large dispersion in pseudo-redshift values obtained. Using the redshift estimated from the Amati relation, a rest-frame lag of $0.048_{-0.015}^{+0.017} \mathrm{~s}$ was determined between 250 and $450 \mathrm{~s}$ post trigger, which, from previously determined trends (Norris et al. 2000) corresponds a peak luminosity of $0.22_{-0.07}^{+0.11} \times 10^{53} \mathrm{erg} \mathrm{s}^{-1}$. Barkov \& Bisnovatyi-Kogan (2005) suggest that the infrared afterglow is the result of dust re-radiation in the envelope surrounding the GRB source and using this model they obtain a lower value of the redshift $\leq 0.12$.

All the indirect redshift, energy and luminosity indicators imply that GRB 041219a is a very luminous burst with isotropic output of about $\sim 5 \times 10^{52} \mathrm{erg}$. It should be noted that the value of $\alpha, E_{\text {peak }}$ and the lag vary throughout this well observed burst, particularly between the precursor and the main emission phase.

\subsection{Broadband spectra}

The broadband spectra of the precursor pulse and the weak pulse at $\sim 60 \mathrm{~s}$ in the long quiescent interval are given in Fig. 4 . The ASM observations (Fig. 2) start at the end of the precursor pulse. The BAT and SPI data show that the spectrum of the precursor peaked at about $2 \times 10^{19} \mathrm{~Hz}(\sim 83 \mathrm{keV})$ and is quite different from the broadband spectra in the two subsequent intervals from 8 to $65 \mathrm{~s}$ and 66 to $120 \mathrm{~s}$ where there is strong emission in the $\mathrm{X}$-ray band. The dominance of the emission in the X-ray band is apparent in the ASM and BAT profiles in Fig. 2. 


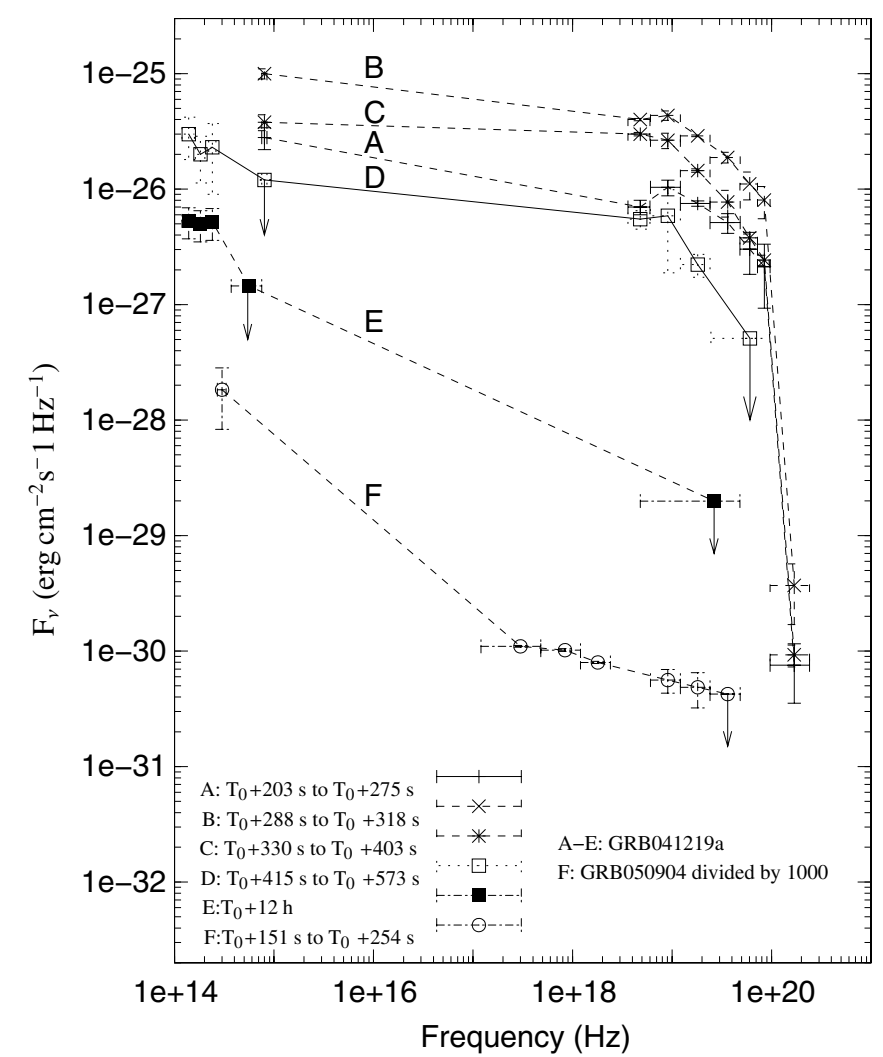

Fig. 6. Broadband spectra of GRB 041219a (A to E) and GRB 050904 (F) at a redshift of 6.29. The data are plotted in flux density (erg cm $\mathrm{cm}^{-2} \mathrm{~s}^{-1} \mathrm{~Hz}^{-1}$ ) versus observed frequency. The four intervals (A to D) are defined by the periods of simultaneous RAPTOR optical observations of GRB 041219a (Vestrand et al. 2005). The RAPTOR optical results, corrected for extinction, are shown and connected by a line to the lowest energy BAT channel (15-25 keV) and the higher energy SPI channels. The PARITEL near infrared results (Blake et al. 2005) are shown for interval D and connect by a solid line with the optical upper limit and the $\gamma$-ray data. The broadband spectrum of the afterglow from GRB 041219a is shown $12 \mathrm{~h}$ after the burst (E) using infrared and optical data from Blake et al. (2005) and Sonoda et al. (2004) respectively and and an upper limit from the afterglow search with SPI. The broadband spectra show the evolution from the peak of the burst in B through the subsequent intervals (C and D) and into the afterglow. The simultaneous optical, X-ray (Boër et al. 2006) and $\gamma$-ray results (this work) for the high redshift GRB 050904 are shown for the interval 151 to $254 \mathrm{~s}$. The data for GRB 050904 have been divided by $10^{3}$ for clarity of presentation and unlike GRB 041219a, GRB 050904 clearly shows the large difference between the optical and $\gamma$-ray emission.

In contrast the broadband spectra over the four intervals (A to D in Fig. 6) are remarkably similar because the simultaneous optical emission is correlated with the gamma ray burst. This correlation would arise naturally if the emission in both bands were generated by a common mechanism. The most likely mechanism is that the optical emission is the low energy tail of the synchrotron radiation generated by internal shocks in the outflow (e.g. Katz 1994; Mészáros \& Rees 1999). Fan et al. (2005) and Fan \& Wei (2004) have suggested that the optical/near IR flash could be the result of emission from neutron-rich internal shocks colliding at a larger distance from the central engine. There are simultaneous $J, H$ and $K$ infrared observations in interval D that are an extrapolation of the optical data. The $J, H$ and $K$-band observations $12 \mathrm{~h}$ after the burst have about the same slope (E in Fig. 6) are similar but less intense and mark the transition from the burst to the afterglow. This similarity between the two measurements indicate that the infrared emission is due to the afterglow and it was already well developed in interval D and has been well modelled as the superposition of a reverse shock and a forward shock component (Fan et al. 2005).

There is an interesting contrast between the broadband spectra of GRB 041219a and GRB 050904 (F in Fig. 6) because of the large difference between the simultaneous optical and $\gamma$-ray emission in the two bursts. The five GRBs that have been detected as strong optical sources during the prompt $\gamma$-ray emission are GRB 990123 at $z=1.60$ (Akerlof et al. 1999), GRB 041219a with no spectroscopic redshift, GRB 050401 at $z=2.9$ (Rykoff et al. 2005), GRB 050904 at $z=6.29$ (Boër et al. 2006; Watson et al. 2006; Tagliaferri et al. 2005; Cusumano et al. 2005; Wei et al. 2005) and GRB 0601111b with no spectroscopic redshift (Klotz et al. 2006). The flux densities at the maximum of the optical emission are respectively $1080 \mathrm{mJy}(V-$ band), $3.1 \mathrm{mJy}$ ( $R$-band), $2.6 \mathrm{mJy}$ ( $R$-band), $1300 \mathrm{mJy}$ ( $V$-band) and $\sim 360 \mathrm{mJy}$ (clear filter) if it is assumed that all GRBs are at the same redshift of 1.6 for GRB 990123 (Boër et al. 2006). At $z=1.6$ the optical emission from GRB 990123, GRB 050904 and GRB 0601111b are comparable and exceed that from GRB 041219a and GRB 050401 by a factor of several hundred. The bright optical emission from GRB 990123, GRB 050904 and GRB 0601111b has been attributed to the reverse shock. This powerful emission is not dominant in GRB 041219a and GRB 050401 because it would have overwhelmed the observed emission. Furthermore the strong optical emission from the reverse shock is not a common feature of most GRBs (Roming et al. 2005). In the additional case of GRB 060124 at a redshift of 2.297 (Mirabal \& Halpern 2006a; Cenko et al. 2006; Prochaska et al. 2006) recent simultaneous optical and $\gamma$-ray observations with Swift reveal prompt optical emission with a peak of 16.88 magnitude in the $V$-band $(0.55 \mathrm{mJy})$ (Holland et al. 2006b). The UVOT instrument was in image mode at early times and high time resolution data was not available. Romano et al. (2006) rule out a reverse shock based on the level of the optical emission and furthermore, since the optical data do not clearly track the $\gamma$-ray emission, they suggest that external shocks may be the source of the optical emission. The long duration and nearby $(z=0.0331$, Mirabal \& Halpern 2006b) weak burst/X-ray flash GRB 060218/SN 2006aj has simultaneous optical and $\gamma$-ray observations with Swift (Cusumano et al. 2006; Campana et al. 2006). The peak of the prompt optical emission is only $0.118 \mathrm{mJy}$ in the $V$ band (Campana et al. 2006) and is smaller than the bright optical emission from GRB 990123 by a factor of $\sim 10^{8}$ (if both bursts were at a redshift of $z=1.6$ ) yielding a much larger ratio in prompt optical than in the prompt $\gamma$-ray emission.

These results underline the need for continued broadband observations of the prompt emission phase and the early afterglow.

\section{Conclusions}

GRB 041219a is the brightest burst localised by INTEGRAL and has a peak flux ( $20 \mathrm{keV}-8 \mathrm{MeV}, 1 \mathrm{~s}$ integration) of $43 \mathrm{ph} \mathrm{cm}^{-2} \mathrm{~s}^{-1}$ $\left(1.84 \times 10^{-5} \mathrm{erg} \mathrm{cm}^{-2} \mathrm{~s}^{-1}\right)$, which places it among the brightest bursts. Furthermore, the $T_{90}$ duration of GRB $041219 \mathrm{a}$ is $186 \mathrm{~s}$ $(\sim 20 \mathrm{keV}-8 \mathrm{MeV})$ making it longer than all but a small number of BATSE bursts. The main burst occurred after a long quiescent interval of $\sim 250 \mathrm{~s}$ which enabled optical and near infrared telescopes to observe the burst while the prompt event was still in progress.

We have presented comprehensive results on the temporal and spectral analyses of GRB 041219a (Figs. 1, 3), including line and afterglow searches using the high resolution 
Germanium spectrometer, SPI, aboard INTEGRAL. Spectra for the burst and sub-intervals were fit by the Band model and also by the quasithermal model (Tables 1 and 2). The high resolution Germanium spectrometer data were searched for emission and absorption features and for $\gamma$-ray afterglow. No significant emission or absorption features were found and limits of $900 \mathrm{eV}$ and $120 \mathrm{eV}$ are set on the most significant features. No $\gamma$-ray afterglow was detected from the end of the prompt phase up to $\sim 12 \mathrm{~h}$ post-burst (Fig. 4). The spectral lag was determined using data from the BAT and changes throughout the burst (Table 1).

We availed of public data from BAT on Swift and obtained ASM observations of Rossi X-ray Timing Explorer (Fig. 2), optical and near-infrared observations published by Vestrand et al. (2005) and Blake et al. (2005). Broadband spectra during 7 intervals in the prompt phase are presented (Figs. 5 and 6) and compared to the high-redshift GRB 050904. The $\gamma$-ray and optical data in GRB 041219a are correlated in contrast with the large difference between the simultaneous optical and $\gamma$-ray emission in GRB 050904.

The results presented here highlight the need for continued broadband observations of $\gamma$-ray burst and the afterglow.

Acknowledgements. S.M.B. acknowledges the support of the European Union through a Marie Curie Intra-European Fellowship within the Sixth Framework Program.

\section{References}

Akerlof, C., Balsano, R., Barthelemy, S., et al. 1999, Nature, 398, 400

Amati, L., Frontera, F., Tavani, M., et al. 2002, A\&A, 390, 81

Atteia, J.-L. 2003, A\&A, 407, L1

Band, D., Matteson, J., Ford, L., et al. 1993, ApJ, 413, 281

Band, D. L., Ford, L. A., Matteson, J. L., et al. 1995, ApJ, 447, 289

Barkov, M. V., \& Bisnovatyi-Kogan, G. S. 2005 [arXiv: astro-ph/0503414]

Barthelmy, S., Burrows, D., Cummings, J., et al. 2004, GCN, 2874

Blake, C. H., Bloom, J. S., Starr, D. L., et al. 2005, Nature, 435, 181

Boër, M., Atteia, J. L., Damerdji, Y., et al. 2006, ApJ, 638, L71

Bosnjak, Z., Celotti, A., \& Ghirlanda, G. 2006 [arXiv: astro-ph/0604425]

Böttcher, M. 2004, Adv. Space Res., 34, 2696

Briggs, M. S., Pendleton, G. N., Brainerd, J. J., et al. 1998, in American Institute of Physics Conference Series, ed. C. A. Meegan, R. D., Preece, \& T. M. Koshut, 104

Campana, S., Mangano, V., Blustin, A. J., Brown, P., \& Burrows, D. N. 2006 [arXiv: astro-ph/0603279]

Cenko, S. B., Berger, E., \& Cohen, J. 2006, GRB Coordinates Network, 4592, 1

Chen, L., Lou, Y.-Q., Wu, M., et al. 2005, ApJ, 619, 983

Costa, E., Frontera, F., Heise, J., et al. 1997, Nature, 387, 783

Crider, A., Liang, E. P., Smith, I. A., et al. 1997, ApJ, 479, L39

Cusumano, G., Barthelmy, S., Gehrels, N., et al. 2006, GRB Coordinates Network, 4775, 1

Cusumano, G., Mangano, V., Chincarini, G., et al. 2005 [arXiv: astro-ph/0509737]

Diehl, R., Baby, N., Beckmann, V., et al. 2003, A\&A, 411, L117

Drago, A., \& Pagliara, G. 2005 [arXiv: astro-ph/0512602]

Fan, Y. Z., \& Wei, D. M. 2004, ApJ, 615, L69

Fan, Y. Z., Zhang, B., \& Wei, D. M. 2005, ApJ, 628, L25

Fenimore, E., Barthelmy, S., Cummings, J., et al. 2004, GCN, 2906

Fishman, G. J., Meegan, C. A., Wilson, R. B., et al. 1994, ApJS, 92, 229

Gehrels, N., Chincarini, G., Giommi, P., et al. 2004, ApJ, 611, 1005

Ghirlanda, G., Celotti, A., \& Ghisellini, G. 2002, A\&A, 393, 409

Ghirlanda, G., Celotti, A., \& Ghisellini, G. 2003, A\&A, 406, 879

Ghirlanda, G., Ghisellini, G., \& Lazzati, D. 2004, ApJ, 616, 331

Golenetskii, S., Aptekar, R., Mazets, E., et al. 2006, GRB Coordinates Network, 4599, 1

Götz, D., Mereghetti, S., Shaw, S., et al. 2004, GCN, 2866

Hakkila, J., \& Giblin, T. W. 2004, ApJ, 610, 361

Hjorth, J., Sollerman, J., Møller, P., et al. 2003, Nature, 423, 847

Holland, S. T., Barthelmy, S., Burrows, D. N., et al. 2006a, GRB Coordinates Network, 4570, 1

Holland, S. T., Smith, P., Huckle, H., \& Gehrels, N. 2006b, GRB Coordinates Network, 4580, 1

Kaneko, Y., Preece, R. D., \& Briggs, M. S. 2003, Am. Astron. Soc. Meet. Abstr., 203
Katz, J. I. 1994, ApJ, 432, L107

Klotz, A., Gentre, B., Stratta, G., et al. 2006 [arXiv: astro-ph/0604061]

Kocevski, D., \& Liang, E. 2003, ApJ, 594, 385

Kocevski, D., Ryde, F., \& Liang, E. 2003, ApJ, 596, 389

Koshut, T. M., Kouveliotou, C., Paciesas, W. S., et al. 1995, ApJ, 452, 145 Lazzati, D. 2005, MNRAS, 357, 722

Lazzati, D., Ghisellini, G., Celotti, A., \& Rees, M. J. 2000, ApJ, 529, L17

Levine, A., \& Remillard, R. 2004, GRB Coordinates Network, 2917, 1

Levine, A. M., Bradt, H., Cui, W., et al. 1996, ApJ, 469, L33

Liang, E. P. 1997, ApJ, 491, L15

Lloyd, N. M., \& Petrosian, V. 2000, ApJ, 543, 722

Lloyd-Ronning, N. M. \& Ramirez-Ruiz, E. 2002, ApJ, 576, 101

Mazets, E. P., Golenetskii, S. V., Aptekar, R. L., Guryan, Y. A., \& Ilinskii, V. N. 1980, Sov. Astron. Lett., 6, 372

McBreen, S., McBreen, B., Hanlon, L., \& Quilligan, F. 2002a, A\&A, 393, L29

McBreen, S., McBreen, B., Quilligan, F., \& Hanlon, L. 2002b, A\&A, 385, L19

McGlynn, S., McBreen, S., Hanlon, L., et al. 2005

[arXiv:astro-ph/0505349]

Medvedev, M. V. 2000, ApJ, 540, 704

Mereghetti, S., Götz, D., Borkowski, J., Walter, R., \& Pedersen, H. 2003, A\&A, 411, L291

Mészáros, P., \& Rees, M. J. 1999, MNRAS, 306, L39

Mészáros, P., \& Rees, M. J. 2000, ApJ, 530, 292

Mirabal, N., \& Halpern, J. P. 2006a, GRB Coordinates Network, 4591, 1

Mirabal, N., \& Halpern, J. P. 2006b, GRB Coordinates Network, 4792, 1

Montroll, E. W., \& Shlesinger, M. F. 1982, Proc. of the National Academy of Sciences of the United States of America-Physical Sciences, 79, 3380

Moran, L., Mereghetti, S., Götz, D., et al. 2005, A\&A, 432, 467

Murakami, T., Fujii, M., Hayashida, K., Itoh, M., \& Nishimura, J. 1988, Nature, 335,234

Murakami, T., Inoue, H., Nishimura, J., van Paradijs, J., \& Fenimore, E. E. 1991, Nature, 350, 592

Nakar, E., \& Piran, T. 2002, ApJ, 572, L139

Norris, J. P., Marani, G. F., \& Bonnell, J. T. 2000, ApJ, 534, 248

Paciesas, W. S., Meegan, C. A., Pendleton, G. N., et al. 1999, ApJS, 122, 465

Page, M., Burrows, D., Beardmore, A., et al. 2005, GRB Coordinates Network, 3830,1

Pal'Shin, V., \& Frederiks, D. 2005, GRB Coordinates Network, 3852, 1

Pelangeon, A., Atteia, J. L., Lamb, D. Q., \& Ricker, G. R. 2005 [arXiv:astro-ph/0601150]

Piran, T. 2004, Rev. Mod. Phys., 76, 1143

Preece, R. D., Briggs, M. S., Mallozzi, R., et al. 2000, ApJS, 126, 19

Preece, R. D., Briggs, M. S., Giblin, T. W., et al. 2002, ApJ, 581, 1248

Prochaska, J. X., Foley, R., Tran, H., Bloom, J. S., \& Chen, H.-W. 2006, GRB Coordinates Network, 4593, 1

Protassov, R., van Dyk, D. A., Connors, A., Kashyap, V. L., \& Siemiginowska, A. 2002, ApJ, 571, 545

Quilligan, F., McBreen, B., Hanlon, L., et al. 2002, A\&A, 385, 377

Ramirez-Ruiz, E., \& Merloni, A. 2001, MNRAS, 320, L25

Ramirez-Ruiz, E., Merloni, A., \& Rees, M. J. 2001, MNRAS, 324, 1147

Ramirez-Ruiz, E., MacFadyen, A. I., \& Lazzati, D. 2002, MNRAS, 331, 197

Rees, M. J., \& Mészáros, P. 2005, ApJ, 628, 847

Romano, P., Campana, S., Chincarini, G., et al. 2006

[arXiv:astro-ph/0602497]

Roming, P. W. A., Schady, P., Fox, D. B., et al. 2005

[arXiv:astro-ph/0509273]

Ryde, F. 2004, ApJ, 614, 827

Ryde, F. 2005a, A\&A, 429, 869

Ryde, F. 2005b, ApJ, 625, L95

Ryde, F., Kocevski, D., Bagoly, Z., Ryde, N., \& Mészáros, A. 2005, A\&A, 432, 105

Rykoff, E. S., Yost, S. A., Krimm, H. A., et al. 2005, ApJ, 631, L121

Sazonov, S. Y., Lutovinov, A. A., \& Sunyaev, R. A. 2004, Nature, 430, 646

Soderberg, A. M., \& Frail, D. A. 2004, GCN, 2881

Sonoda, E., Maeno, S., Matsuo, Y., \& Yamauchi, M. 2004, GRB Coordinates Network, 2882

Tagliaferri, G., Antonelli, L. A., Chincarini, G., et al. 2005, A\&A, 443, L1

van der Horst, A. J., Rol, E., \& Strom, R. 2004a, GCN, 2894

van der Horst, A. J., Rol, E., \& Strom, R. 2004b, GCN, 2895

Vanderspek, R., Sakamoto, T., Barraud, C., et al. 2004, ApJ, 617, 1251

Vedrenne, G., Roques, J. P., Schönfelder, V., et al. 2003, A\&A, 411, L63

Vestrand, W. T., Wozniak, P. R., Wren, J. A., et al. 2005, Nature, 435, 178

Watson, D., Hjorth, J., Levan, A., et al. 2004, ApJ, 605, L101

Watson, D., Reeves, J. N., Hjorth, J., et al. 2006, ApJ, 637, L69

Wei, D. M., Yan, T., \& Fan, Y. 2005 [arXiv: astro-ph/0511154]

Winkler, C., Courvoisier, T. J. L., Di Cocco, G., et al. 2003, A\&A, 411, L1

Yonetoku, D., Murakami, T., Nakamura, T., et al. 2004, ApJ, 609, 935

Zhang, B., \& Mészáros, P. 2004, Int. J. Mod. Phys. A, 19, 2385 